\title{
Effects of a Short Course of Oral Prednisolone in Patients with Bladder Pain Syndrome with Fluctuating, Worsening Pain despite Low-Dose Triple Therapy
}

\author{
Hee Jong Jeong \\ Department of Urology, Wonkwang University School of Medicine \& Hospital, Iksan, Korea
}

\begin{abstract}
Purpose: Triple therapy with gabapentin, amitriptyline, and nonsteroidal antiinflammatory drugs is efficacious for chronic bladder pain syndrome/interstitial cystitis (BPS/IC). However, transient, fluctuating, worsening pain or flare-up symptoms may develop during treatment for a variety of reasons. Here, we assessed the validity of our observational experience regarding a short course of oral prednisolone therapy, which might be of value in the management of flare-up symptoms of BPS/IC.

Methods: Between May 2007 and May 2012, 7 women (mean age, 61.5 years; range, 44.8 to 75.4 years) with BPS/IC presenting with transient, fluctuating, worsening pain as a flare-up symptom despite low-dose triple therapy received a 1- to 3-month course of oral prednisolone $10 \mathrm{mg}$. The outcome measures used were the IC symptom scale (ICSS, O'Leary-Sant Interstitial Cystitis Symptom Index) and a visual analogue scale (VAS), which were completed at baseline and after treatment.

Results: There were statistically significant differences in the ICSS and VAS score before and after prednisolone treatment $(\mathrm{P}<0.05$ by Wilcoxon singed-rank test). The pretreatment IC symptom index (ICSI), IC problem index (ICPI), and VAS score were $16.7 \pm$ $2.2,13.7 \pm 2.3$, and $8.3 \pm 1.5$ (mean \pm standard deviation $[S D]$ ), and the posttreatment scores were $4.9 \pm 2.3,4.3 \pm 1.1$, and $2.5 \pm 0.9$ $($ mean \pm SD), respectively. The ICSI, ICPI, and VAS scores were improved after prednisolone treatment by $70.7 \%, 68.6 \%$, and $69.9 \%$, respectively. Low-dose triple therapy with prednisolone caused no significant adverse effects.

Conclusions: In patients with BPS/IC who show transient, fluctuating, worsening pain as flare-up symptoms despite undergoing low-dose triple therapy, a short course of oral prednisolone therapy was sufficiently effective. However, large-scale studies should be performed to verify our findings.
\end{abstract}

Keywords: Interstitial cystitis; Pain measurement; Adrenal cortex hormones

\section{INTRODUCTION}

Bladder pain syndrome/interstitial cystitis (BPS/IC) is defined as unpleasant sensations such as pain, pressure, and discomfort of the bladder and the lower urinary tract, with symptoms lasting for 6 weeks or longer in the absence of infection or any other confirmed causes [1-3]. The prevalence of BPS/IC varies greatly by nation, race, and definition, but the occurrence rate is approximately $0.3 \%$, with the incidence being 10 to $20 \%$ lower among men than women. However, in some population-based studies that used questionnaires to collect patient information,
BPS/IC was 30 to 50 times more frequent [2].

The etiology of BPS is more complex, and basic theories abound regarding its pathogenesis, involving leaky epithelium, mast cell activation, neurogenic inflammation, or some combinations of these and other factors. Investigators should explore the central neurological mechanisms of pathogenesis, as well as genetic/familial, immunological, and infectious etiologies of this puzzling, complex disorder. Irritable bowel syndrome, fibromyalgia, chronic fatigue syndrome, and various other chronic pain disorders may also be associated with BPS in some patients [1-3].
Corresponding author: Hee Jong Jeong

Department of Urology, Wonkwang University School of Medicine \& Hospital, 895 Muwang-ro, Iksan 570-974, Korea

Tel: +82-63-859-1332, Fax: +82-63-842-1455, E-mail: uro94c@wku.ac.kr

Submitted: November 19, 2012 / Accepted after revision: December 24, 2012
This is an Open Access article distributed under the terms of the Creative Commons Attribution Non-Commercial License (http://creativecommons.org/licenses/by-nc/3.0/) which permits unrestricted non-commercial use, distribution, and reproduction in any medium, provided the original work is properly cited. 
It is important to reassure the patient that such symptoms, although bothersome, are not signs of a life-threatening disease. In the absence of disease progression, treatment should be focused on reducing symptom severity. Some patients get better naturally and even heal completely within a few weeks or months [2]. Therefore, the most widely and effectively used treatments are conservative treatments such as patient education, empowerment, dietary manipulation, pelvic floor relaxation, pain killers including narcotic analgesics, tricyclic antidepressants, anticonvulsants, nonsteroidal antiinflammatory drugs (NSAIDs), and intravesical therapy, either singly or in combination. However, when such conservative treatments are hardly or not effective, cystoscopy under anesthesia with bladder hydrodistention, fulguration of Hunner lesion, intramural botulinum toxin injection, neuromodulation, diversion with or without cystectomy, and substitution cystoplasty can be applied [2-4].

Despite such treatments, some patients often show transient, fluctuating, worsening pain or flare-up symptoms for unknown reasons, which make them feel hopeless about their treatment. Furthermore, the doctor-patient relationship is destroyed, leading to treatment failure. Because BPS/IC is associated with immunological, inflammatory diseases such as allergies, fibromyalgia, vulvodynia, inflammatory bowel disease, systemic lupus erythematosus, focal vulvitis/vulvar vestibulitis, and Sjögren's syndrome, corticosteroids having antiinflammatory and immune-modifying properties have the potential for use in BPS/ IC treatment [2,3]. In a few case reports on IC associated with Sjögren's syndrome, corticosteroid treatment was effectively used to treat bladder symptoms. Therefore, as a therapy targeting the signal cascades of the immunological system, neurological system, and mast cells, there is a biological plausibility that steroids could be of value in the treatment of this condition $[2,5]$. However, we have yet to find reports on the use of corticosteroids for BPS/IC. Thus, we wanted to verify whether corticosteroid therapy is useful in the treatment of flare-up symptoms in BPS/IC patients undergoing low-dose triple therapy. In the present study, therefore, we assessed the validity of our observational experience of a short course of oral prednisolone (PDS) therapy, which might be of value in the management of flare-up symptoms of BPS/IC.

\section{MATERIALS AND METHODS}

Between May 2007 and May 2012, 7 women (mean age, 61.5 years; range, 44.8 to 75.4 years) with BPS/IC who showed tran- sient, fluctuating, worsening pain as flare-up symptoms despite undergoing low-dose triple therapy were included in the present study. We limited the definition of flare-up symptoms to symptoms that were increased by over $50 \%$ in terms of the International Cystitis Symptom Index (ICSI), International Cystitis Problem Index (ICPI), and visual analogue scale (VAS) scores owing to breakthrough pain for 2 weeks or longer among patients who had been receiving low-dose triple therapy for at least 3 months. In addition, we only performed PDS treatment when patients with flare-up symptoms did not show improvement after using 2 to 3 times the amitriptyline and gabapentin dose (maximum doses: amitriptyline, $75 \mathrm{mg}$; gabapentin, 900 $\mathrm{mg}$ ) compared with the dose before the occurrence of flare-up symptoms. One hundred five patients were treated with lowdose triple therapy using NSAIDs, amitriptyline, and gabapentin. Among these patients, 7 patients showed transient, fluctuating, worsening pain as a flare-up symptoms. A total of 11 flareup symptoms were observed in these patients, once in four patients, twice in two patients, and thrice in one patient. One of seven patients showed red blood cells in urinalysis and underwent cystoscopy, but there were no special findings other than glomerulation and Hunner's lesion. Although this patient received intravesical chondroitin sulfate installation treatment with triple therapy, the symptoms were not improved.

All patients with flare-up symptoms were prescribed a 1- to 3-month course of oral PDS $10 \mathrm{mg}$, and the dose was tapered to $5 \mathrm{mg}$ and then the medication was suspended once the symptoms were significantly improved.

The outcome measures used were the IC symptom scale (O'Leary-Sant Interstitial Cystitis Symptom Index) and VAS, which were completed at baseline and after treatment. Patients were excluded from the study if there was any contraindication to steroid therapy. We used SPSS ver. 13.0 (SPSS Inc., Chicago, IL, USA) for statistical analysis, and P-values below 0.05 were deemed statistically significant.

\section{RESULTS}

The mean symptom period of the 7 BPS patients with flare-up symptoms before visiting the urology clinic at our hospital was 40.6 months, and low-dose triple therapy had been prescribed for 24.9 months on average. The ICSI, ICPI, and VAS scores were considerably improved after 3 months of low-dose triple therapy (Table 1, Fig. 1).

During treatment of the 7 BPS patients with low-dose triple 
Table 1. Demographic and clinical characteristics of patients according to treatment group

\begin{tabular}{|c|c|c|c|c|}
\hline \multirow{2}{*}{ Characteristic } & \multirow{2}{*}{ Baseline } & \multirow{2}{*}{$\begin{array}{l}\text { 3-month after } \\
\text { low-dose triple therapy }\end{array}$} & \multicolumn{2}{|c|}{ Flare-up symptom $(\mathrm{n}=11)$} \\
\hline & & & Before PDS & After PDS \\
\hline \multicolumn{5}{|l|}{ No. of patients $(n=7)$} \\
\hline Male:female & $0: 7$ & & & \\
\hline Age (yr) & $61.5(44.8-75.4)$ & & & \\
\hline Pretreatment period (mo) & $40.6(4-20)$ & & & \\
\hline Period of low-dose triple therapy (mo) & $24.9(10-60)$ & & & \\
\hline Period of PDS treatment (day) & $47.8(28-51)$ & & & \\
\hline Interstitial cystitis symptom index & $16.9 \pm 3.0(9-9)$ & $9.0 \pm 3.9(3-4)^{\mathrm{a})}$ & $16.7 \pm 2.2(12-20)$ & $4.9 \pm 2.3(2-8)^{\mathrm{a})}$ \\
\hline Interstitial cystitis problem index & $14.3 \pm 2.2(8-6)$ & $5.1 \pm 1.4(3-8)^{\mathrm{a})}$ & $13.7 \pm 2.3(9-6)$ & $4.3 \pm 1.1(3-6)^{\mathrm{a})}$ \\
\hline Visual analogue scale & $8.0 \pm 1.6(4-0)$ & $4.0 \pm 0.9(3-5)^{\mathrm{a})}$ & $8.3 \pm 1.5(5-0)$ & $2.5 \pm 0.9(1-4)^{\mathrm{a})}$ \\
\hline
\end{tabular}

Values are presented as mean (range) or mean \pm standard deviation (range).

PDS, prednisolone.

${ }^{a)} \mathrm{P}<0.05$ by Wilcoxon singed-rank test.

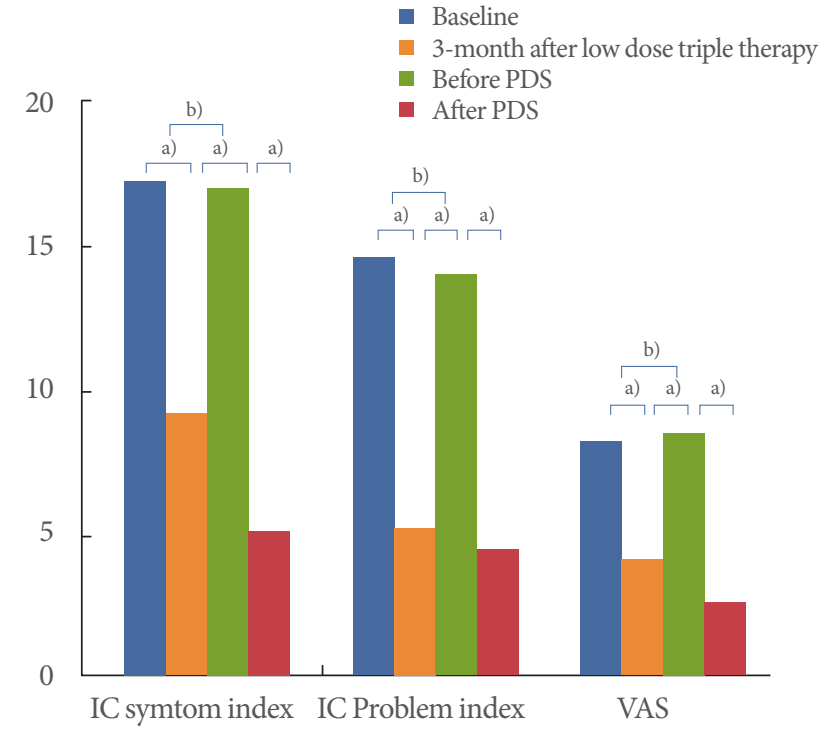

Fig. 1. Changes in flare-up symptoms from before to after prednisolone (PDS) treatment. IC, interstitial cystitis; VAS, visual analogue scale. ${ }^{\text {a }} \mathrm{P}<0.05$ and ${ }^{\mathrm{b})} \mathrm{P}>0.05$ by Wilcoxon singed-rank test.

therapy, 11 flare-up symptoms were detected. The mean PDS treatment period for flare-up symptoms was 47.8 days, and the symptoms were improved in over $50 \%$ of all patients (Table 1 , Fig. 1).

There were statistically significant differences in the IC symptom scale and VAS score before and after PDS treatment, respectively $(\mathrm{P}<0.05$ by Wilcoxon singed rank test). The pretreatment ICSI, ICPI, and VAS scores were $16.7 \pm 2.2,13.7 \pm 2.3$, and
$8.3 \pm 1.5$ (mean \pm stnadard deviation $[\mathrm{SD}]$ ), and the posttreatment scores were $4.9 \pm 2.3,4.3 \pm 1.1$, and $2.5 \pm 0.9$ (mean $\pm \mathrm{SD}$ ), respectively. The ICSI, ICPI, and VAS scores improved after PDS treatment by $70.7 \%, 68.6 \%$, and $69.9 \%$, respectively, compared with those before PDS treatment. A short course of oral PDS with low-dose triple therapy caused no significant adverse effects (Table 1, Fig. 1).

\section{DISCUSSION}

BPS/IC is a clinical diagnosis based primarily on chronic symptoms of pain perceived by the patient to emanate from the bladder or pelvis associated with urinary urgency and frequency in the absence of other identified causes of the symptoms [2]. The prevalence of chronic pain due to benign causes, including BPS/ IC, is reported to be at least $10 \%$ in the general population $[3,4]$. Pain is defined as an unpleasant sensory and emotional experience associated with actual or potential tissue damage or an experience described in terms of such damage. Pain can be categorized as physiologic pain of the normal state and pathophysiologic pain of the abnormal state. Physiologic pain represents nociceptive pain that disappears as soon as the nociception input disappears. Pathophysiologic pain continuously occurs, even after the nociception input disappears or the tissue damage is repaired. Pathophysiologic pain can be further divided into nociceptive pain, neuropathic pain, and psychogenic pain; pathophysiologic pain causes spontaneous pain, evoked pain, etc. Pathophysiologic pain is not a warning sign, but is a pathological 
condition that harms the body, particularly if inflammation or damage occurs around or in the nerve tissues [1-4].

Continual or repetitive trauma, toxins, infection, or inflammation affecting the pelvis or bladder in BPS/IC patients can lead to peripheral nerve stimulation or damage, which is well known as pathophysiologic nociceptive pain. Neuropathic pain refers to pain arising as a result of damage to any part of the sensory system. Neuromas, regenerating sprouts, and demyelination are thought to be responsible for the ectopic discharge of injured nerves. In contrast with nociceptive pain, the quality of pain associated with neuropathic lesions is often entirely new and different to the patient. Specific emotional factors such as anxiety and depression are known to enhance the experience of pain, which can be categorized as psychogenic pain. Adrenaline and carbon dioxide during anxiety and stressful periods increase the activities of the reticular activating system in the midbrain and rostral pons and the transmission of nociceptive input to the thalamus and the cerebral cortex. Depression can enhance nociception by reducing the activity of the powerful descending inhibitory pathways from the brainstem. Anxiety or depression can strengthen the pain, whereas chronic pain induces even more stress in the depressed patient, leading to aggravation of the condition $[1,6,7]$.

Peripheral sensitization of primary afferent sensory fibers by mediators of the pain response results in greater and more frequent transmission of action potentials to nociceptive neurons than in "normal" pain responses, and central sensitization can also cause an individual to perceive greater and more prolonged pain owing to increased central neuronal responsiveness. Pathophysiologic pain has characteristic features including hyperexcitation, receptive field expansion, hyperalgesia, allodynia caused by long-term changes in nociceptive neurons, peripheral or central sensitization, and wind-up. Thus, BPS/IC patients have simple bladder pain as well as symptoms like hyperalgesia and allodynia in their complicated or amplified form. This "pain memory" in the central nervous system may be why IC patients become refractory to different therapies $[1,8,9]$.

In the treatment of BPS/IC, patient education and empowerment, dietary manipulation, pelvic floor relaxation, pain killers including narcotic analgesics, intravesical therapy, intramural botulinum toxin injection, neuromodulation, and surgical intervention have been adopted with variable success $[1,2]$. Despite the diversity of treatment, BPS/IC is a chronic disease, and no current treatment has a significant impact on symptoms over time in the majority of patients. If bladder inflammation by a progressive defect in epithelial permeability were suspected as the cause of the bladder pain in BPS/IC patients, the epithelial defect, bladder inflammation, and bladder pain should be selected as targets of treatment. However, if bladder inflammation by epithelial defect does not currently exist but was the cause of the current pain owing to its existence at a specific time in the past, BPS/IC must be considered as a chronic pain syndrome. In such a case, treatment must be focused on effective, quick, and long-term removal of pain.

The basic principles of pain treatment are as follows. First, pain must be quickly treated before peripheral or central sensitization; prognosis can be improved by faster treatment. Second, if pain remains after various treatment modalities, treatment must be continued for a longer period to reduce or remove the pain. Third, all parts of the pain development mechanism, from direct injury or inflammation to the nerves in the peripheral or central nervous system and peripheral nociception to central sensitization, must be targets of pain treatment by use of diverse drugs.

We performed low-dose triple therapy until bladder pain was relieved in BPS/IC patients. However, despite such continued treatment, we observed breakthrough, exacerbation, or periodic flare-up symptoms with transient, fluctuating, worsening characteristics in 7 patients. These patients were prescribed PDS 10 $\mathrm{mg}$, once a day for 1 to 3 months. Once the flare-up symptoms disappeared, PDS was slowly stopped after tapering the dose, and only triple therapy was continued.

Our triple therapy comprised minimal doses of each drug, including tricyclic antidepressants (amitriptyline, duloxetine), anticonvulsants (gabapentin, pregabalin), and analgesics (NSAIDs), and we then compared the scores before and after low-dose triple therapy. The ICSI and ICPI scores at 1, 3, and 6 months after low-dose triple therapy showed a 60 to $70 \%$ improvement, and the VAS score showed an improvement of more than $70 \%$. These improvements were statistically significant. When pain disappears, the drug treatment should be stopped and the patient should be monitored carefully [1].

In patients in whom bladder pain is not improved by lowdose triple therapy, treatment with opioids, neuroleptics, and a2-agonists affecting the central nervous system such as the brain and the spinal cord have been attempted. Such methods have not shown satisfactory effects, however, and are accompanied by many side effects. Most opioids are $\mu$-opioid receptor agonists or drugs with direct affinity for $\mu$-opioid receptors. Shortterm opioid therapy can relieve the breakthrough or exacerbat- 
ed pain or show relapse of pain with an average decrease of approximately $30 \%$. However, the frequent occurrence of significant adverse events such as tolerance, dependence, withdrawal phenomenon, individual variability in response, opioid-induced hyperalgesia, and deleterious effects on quality of life, including mood, disability, activities, and sex, limit the use of these drugs. Also, neuroleptics (haloperidol, chlorpromazine) block dopaminergic receptors at mesolimbic sites, and $\alpha 2$-agonists (clonidine, Catapres) are known to function through activation of the descending inhibitory pathway in the dorsal horn [10-12].

We chose to use corticosteroids in our patients with BPS/IC for the following reasons. Firstly, corticosteroids have antiinflammatory, immune-modifying, and pain-modulating properties that target the immunity, inflammatory, and neurological cascades. IC has many features of an autoimmune diseasechronicity, exacerbation, remission, high prevalence of antinuclear antibodies, and association with other autoimmune syndromes [13]. There have been several reports of patients with lupus cystitis who improved with immunosuppressant therapy including corticosteroids [5]. The most striking effect of glucocorticoids is inhibition of the endoneurial expression of proinflammatory cytokines (cytokines, enzymes, receptors, and adhesion molecules). There are ample evidences that an increase in the level of endoneurial tumor necrosis factor-alpha (TNF- $\alpha$ ) contributes to neuropathic pain following nerve injury. Neuroinflammation is supposed to play a crucial role in the pathology of neuropathic pain [14]. It is conceivable that glucocorticoids might modulate this neuroimmune interaction. Therefore, antiinflammatory and immunosuppressive effects of corticosteroids are indicated to improve pain by inhibiting the production of inflammatory mediators, reducing prostaglandin synthesis, and suppressing ectopic neural discharges from the injured or inflamed fibers [15-17].

Secondly, corticosteroids can work as a mast cell stabilizer to prevent symptoms and the progression of BPS/IC. Mast cells contain vasoactive and inflammatory mediators (e.g., histamine, leukotrienes, prostaglandins, and tryptases) and play a central role in the pathogenesis of neuroinflammatory conditions, including IC. Degranulation occurs in response to immunoglobulin E, substance P, cytokines, bacterial toxins, allergens, toxins, and stress. Mastocytosis occurs in 30 to $65 \%$ of IC patients. Increased levels of histamine, histamine metabolites, and tryptase occur in IC patients. The therapeutic response to treatment with antihistamines (e.g., hydroxyzine) and leukotriene inhibitors speaks to the role of mast cells in IC pathogenesis
[13]. TNF may act directly on mast cells or indirectly by inducing the secretion of mast cell chemokines in neurogenic cystitis. Mast cell activation could then stimulate C-fibers and result in pain. Thus, blockage of mast cell migration to the lamina propria as well as blockage of mast cell activation would minimize mast cell activation of C-fibers. Endoneurial mast cells have a key role in the pain syndromes seen after nerve injury via their release of TNF- $\alpha$ and other mediators, including factors that recruit leukocytes to the site of injury. Glucocorticoid therapy for neuropathic pain may work via the reduced expression of TNF- $\alpha$ in endoneurial mast cells $[18,19]$.

On the basis of this knowledge, we administered oral PDS when the flare-up phenomenon appeared, and successful results were achieved in 7 patients. To the best of our knowledge, this is the first trial to assess such an approach to treatment. Compared with the values before PDS treatment, the ICSI, ICPI, and VAS scores improved after PDS treatment by $70.7 \%, 68.6 \%$, and $69.9 \%$, respectively. Low-dose triple therapy with PDS caused no significant adverse effects including mood change.

Instead of using a double-blind placebo-controlled trial, we used a research model in which the pretreatment pain level of the patient was set as the control. This was done because, first, the use of placebo in patients with severe pain is unethical and patient agreement cannot be obtained easily. Especially with the existence of effective treatment, delay in treatment may cause progression of the disease. Second, the necessity of placebo-controlled trials has been questioned by easy unblinding caused by the side effect profile and drug efficacy in the active arm of the study. The results of the present study are believed to have greater reliability with a higher value compared to $35 \%$, the maximum effect generally anticipated with placebo. In patients with BPS/IC who showed transient, fluctuating, worsening pain as a flare-up symptom despite undergoing low-dose triple therapy, a short course of oral PDS therapy was sufficiently effective. However, large-scale studies should be performed to verify our findings.

\section{CONFLICT OF INTEREST}

No potential conflict of interest relevant to this article was reported.

\section{ACKNOWLEDGEMENTS}

This work was supported by Wonkwang University in 2012. 


\section{REFERENCES}

1. Lee JW, Han DY, Jeong HJ. Bladder pain syndrome treated with triple therapy with gabapentin, amitriptyline, and a nonsteroidal antiinflammatory drug. Int Neurourol J 2010;14:256-60.

2. Hanno PM. Painful bladder syndrome/interstitial cystitis and related disorders. In: Wein AJ, Kavoussi LR, Novick AC, Partin AW, PetersCA, editors. Campbell-Walsh urology. 10th ed. Philadelphia: Saunders; 2011. p. 357-401.

3. Hanno P, Dmochowski R. Status of international consensus on interstitial cystitis/bladder pain syndrome/painful bladder syndrome: 2008 snapshot. Neurourol Urodyn 2009;28:274-86.

4. Fall M, Baranowski AP, Elneil S, Engeler D, Hughes J, Messelink EJ, et al. EAU guidelines on chronic pelvic pain. Eur Urol 2010;57:3548 .

5. Shibata S, Ubara Y, Sawa N, Tagami T, Hosino J, Yokota M, et al. Severe interstitial cystitis associated with Sjögren's syndrome. Intern Med 2004;43:248-52.

6. Savidge CJ, Slade P. Psychological aspects of chronic pelvic pain. J Psychosom Res 1997;42:433-44.

7. Banks SM, Kerns RD. Explaining high rates of depression in chronic pain: a diathesis-stress framework. Psychol Bull 1996;119:95-110.

8. Gottschalk A, Smith DS. New concepts in acute pain therapy: preemptive analgesia. Am Fam Physician 2001;63:1979-84.

9. de Leon-Casasola O. New developments in the treatment algorithm for peripheral neuropathic pain. Pain Med 2011;12 Suppl 3:S100-8.

10. Nickel JC. Opioids for chronic prostatitis and interstitial cystitis: lessons learned from the 11th World Congress on Pain. Urology
2006;68:697-701.

11. Marmura MJ. Use of dopamine antagonists in treatment of migraine. Curr Treat Options Neurol 2012;14:27-35.

12. Giovannoni MP, Ghelardini C, Vergelli C, Dal Piaz V. Alpha2-agonists as analgesic agents. Med Res Rev 2009;29:339-68.

13. Sant GR. Etiology, pathogenesis, and diagnosis of interstitial cystitis. Rev Urol 2002;4 Suppl 1:S9-15.

14. Minami M, Katayama T, Satoh M. Brain cytokines and chemokines: roles in ischemic injury and pain. J Pharmacol Sci 2006;100:461-70.

15. Tfelt-Hansen P, Daugaard D, Lassen LH, Iversen HK, Olesen J. Prednisolone reduces nitric oxide-induced migraine. Eur J Neurol 2009; 16:1106-11.

16. Eker HE, Cok OY, Aribogan A. A treatment option for post-injection sciatic neuropathy: transsacral block with methylprednisolone. Pain Physician 2010;13:451-6.

17. Bates SM, Hill VA, Anderson JB, Chapple CR, Spence R, Ryan C, et al. A prospective, randomized, double-blind trial to evaluate the role of a short reducing course of oral corticosteroid therapy in the treatment of chronic prostatitis/chronic pelvic pain syndrome. BJU Int 2007;99:355-9.

18. Chen MC, Blunt LW, Pins MR, Klumpp DJ. Tumor necrosis factor promotes differential trafficking of bladder mast cells in neurogenic cystitis. J Urol 2006;175:754-9.

19. Hayashi R, Xiao W, Kawamoto M, Yuge O, Bennett GJ. Systemic glucocorticoid therapy reduces pain and the number of endoneurial tumor necrosis factor-alpha (TNFalpha)-positive mast cells in rats with a painful peripheral neuropathy. J Pharmacol Sci 2008;106: 559-65. 